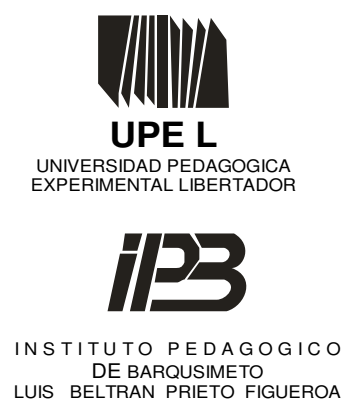

DE BARQUSIMETO
LUIS BELTRAN PRIETO FIGUEROA

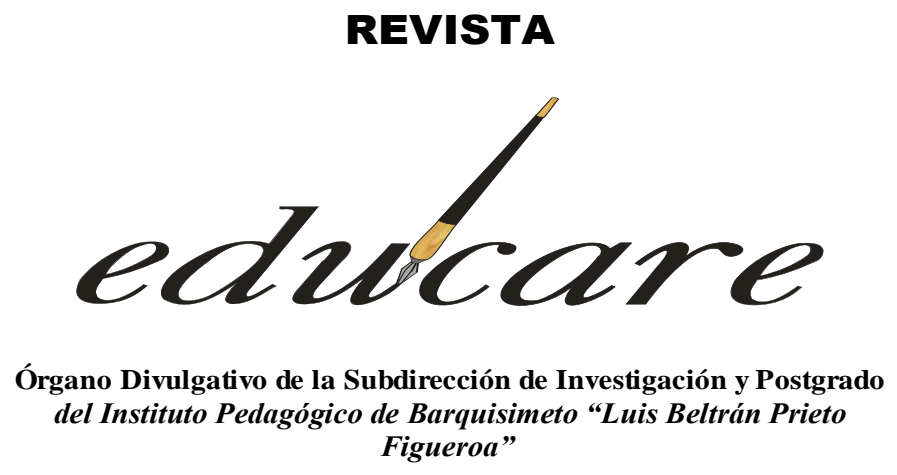

Figueroa"

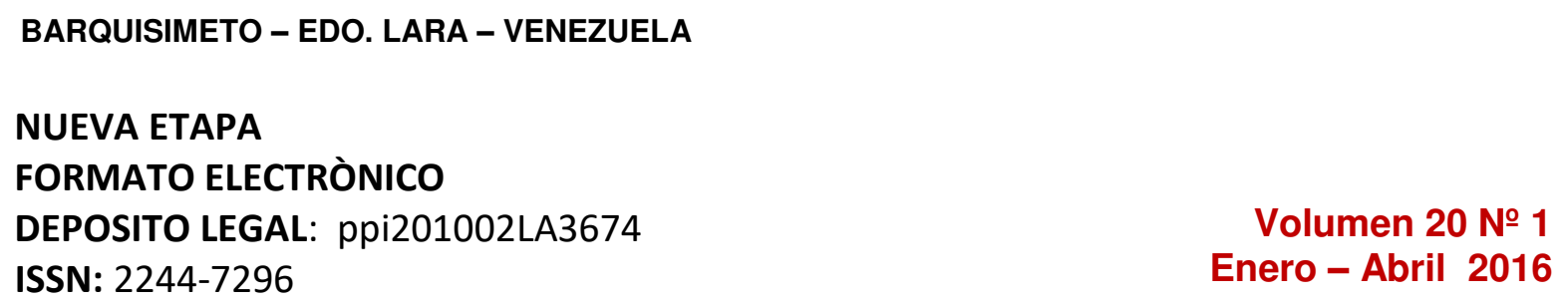

\title{
FACTORES QUE CONTRIBUYEN AL PROCESO DE TUTORIZACIÓN VIRTUAL EN LA UPEL-IPRGR
}

FACTORS CONTRIBUTING TO THE VIRTUAL TUTORING PROCESS AT UPEL-IPRGR

\author{
Andrés Sánchez Rosal *
}

\section{* UNIVERSIDAD PEDAGÓGICA EXPERIMENTAL LIBERTADOR. INSTITUTO PEDAGÓGICO RURAL GERVASIO RUBIO UPEL-IPRGR}




\section{FACTORES QUE CONTRIBUYEN AL PROCESO DE TUTORIZACIÓN VIRTUAL EN LA UPEL-IPRGR}

\section{FACTORS CONTRIBUTING TO THE VIRTUAL TUTORING PROCESS AT UPEL- IPRGR}

\section{TRABAJO DE INVESTIGACIÒN}

Recibido:27-10-15

\section{Andrés Sánchez Rosal * UPEL IPRGR}

Aceptado:11-02-16

\section{RESUMEN}

La presente investigación tiene como propósito establecer los factores que permite la tutorización en línea basado en los referentes teóricos de las funciones y roles del tutor virtual. El estudio tuvo como muestra 77 estudiantes de postgrado que experimentaron su aprendizaje en el aula virtual de la Universidad Pedagógica Experimental Libertador "Gervasio Rubio", Edo Táchira. La investigación en el ámbito metodológico es de naturaleza cuantitativa bajo el nivel descriptivo y se basó en el análisis factorial como técnica estadística, donde se aplicó un cuestionario conformado por 21 ítems. En el estudio se evidenció que el docente de forma regular focaliza las discusiones en los foros de discusión, lo que dificulta la interacción social de los estudiantes durante las actividades educativas en el aula virtual y el aprendizaje colaborativo.

Descriptores: función del tutor virtual, educación en línea, aula virtual.

\begin{abstract}
The present research aims to establish the factors that allow online tutoring based on the theoretical references of the functions and virtual tutor roles. The study had as sample 77 postgraduate students who experienced their learning in the virtual classroom of the Experimental Pedagogical University Libertador "Gervasio Rubio", Táchira State. This is a quantitative research under the descriptive level and was based on the factorial analysis as statistical technique, where a 21 items questionnaire was applied. The study showed that teachers regularly focus discussions in discussion forums, which hinders the social interaction of students during the educational activities in the virtual classroom and collaborative learning.
\end{abstract}

Keywords: Function of virtual tutor, online education, and virtual classroom.

\footnotetext{
*Licenciado en Educación mención Matemática e Informática, Magister en Informática Educativa y Doctor en Educación Experto en Tecnología Educativa. Docente a dedicación tiempo completo en la Universidad Pedagógica Experimental Libertador. Instituto Pedagógico Rural Gervasio Rubio Miembro del Núcleo de Investigación Didáctica y Tecnología Educativa. Correo: andressanchezrosal@gmail.com
} 


\section{INTRODUCCIÓN}

En el mundo educativo continuamente emergen nuevas formas de enseñar y de aprender, y el espacio condicionado por los medios de comunicación social y la tecnologías de información establece nuevas demandas educativas e innovadoras perspectivas de formación educativa, donde es posible la flexibilidad y la autonomía al aprender (García, 2001a) .

Al respecto, la Organización de las Naciones Unidas para la Educación, la Ciencia y la Cultura -Unesco-(1998) y su informe final de la Conferencia Mundial sobre la Educación Superior, reconoce que las tecnologías de información y comunicación permiten renovar la educación superior en la medida que amplíe y diversifique la transmisión del saber, al disponer de las TIC, como ente transmisor del conocimiento y la información a un gran público en general, de forma equitativa y según sus cambiantes intereses.

En consecuencia, los nuevos escenarios educativos plantean una nueva realidad, que según Montserrat y Gisbert (2007) se caracteriza por una creciente movilidad de los estudiantes y la tendencia a establecer novedosas políticas de participación con la emergencia de fomentar sistemas de orientación, seguimiento y evaluación que garanticen la calidad en la formación universitaria abierta y a distancia.

La sociedad actual, caracterizada por el auge de la cibercultura término acuñado por Levy (Citado por Salas, 2012) quien lo define como un conglomerado de técnicas, representaciones y valores propios del ciberespacio, donde los espacios educativos son influenciados por los medios tecnológicos, presenta retos y desafíos a los actores educativos en el surgimiento de sus nuevos roles y funciones, en la cual Pérez (2003) menciona la actual crisis de la modernidad, época desgarrada por una crisis de crecimiento que exige cambios educativos en la figura y concepción del docente.

En las aulas virtuales educativas el docente universitario manifiesta su renovada praxis en ambientes de aprendizaje considerados como alternativa o complemento de la educación tradicional de aula, dentro de los subsistemas de educación superior, ya sea en los cursos impartidos en pregrado o postgrado.

Estos ambientes educativos consideran algunos elementos pedagógicos abordados de igual forma en el aula tradicional, como a través de la dinámica del aprendizaje, al 
tratarse los contenidos desarrollados mediante las estrategias como la discusión, el trabajo colaborativo y cooperativo, acciones necesarias para el análisis y la reflexión del conocimiento generado por una comunidad reunida con fines educativos.

La tutorización virtual implica un proceso de orientación con la finalidad de lograr un conjunto de objetivos educativos en un ambiente técnico-humano donde es posible la aclaración de dudas con el contenido desarrollado, evitando el aislamiento y el posible abandono del estudiante, en su participación en el aula virtual (Llorente, 2006).

Ahora bien, el proceso de tutorización, requiere que los estudiantes sean asistidos de manera apropiada por quien conduce la organización y la planificación de todas las actividades, para el logro de las metas educativas, cuya práctica docente está integrada por un cúmulo de acciones, y de forma análoga al aula tradicional, conducen al éxito o el fracaso de la práctica pedagógica.

En ese sentido, el término tutorización está ampliamente definido por un conjunto de autores avocados al estudio de este concepto, acepción que proviene de la formación virtual , y en el primer orden se destaca Monserrat y Gisbert (Op. cit) quienes subrayan su definición como una acción tutorial, señalada netamente como un proceso formativo y orientador, dirigido al estudiante, siempre en búsqueda del crecimiento profesional y centrada en la atención individual durante la integración de las diversas áreas de conocimiento.

En otro orden de ideas, aunque el término tutorización o tutor virtual engloba de por sí la educación a distancia, según Seone., García. , Bosom, Fernández y Hernández (2006) la tutoría virtual requiere paradójicamente de una fuerte presencialidad por parte del tutor, donde es importante su intervención profesional en el diseño de las actividades, el seguimiento continuo de los aprendizajes y la posterior evaluación de las competencias solicitadas

Aun cuando la presencialidad del tutor se destaca por sí misma en las características que dispone el aula virtual, esto también implica de forma sucinta desde la presentación de los contenidos hasta la calidad de las actividades formativas, con su respectivo y necesario seguimiento.

Ahora en cuanto a el rol del tutor virtual, según Medina, Rico y Rico (2011) se 
establece como asesor, comunicador, motivador e instructor, y dentro de su función pedagógica es importante su participación activa en el seguimiento a nivel individual y grupal de los estudiantes con respecto a las actividades realizadas dentro del aula virtual.

En consecuencia, el aprendizaje en un aula virtual depende en gran manera de la actuación del docente de acuerdo a su formación como tutor virtual de forma natural, según sus habilidades naturales y sociales, o según las prescripciones que desde el fundamento teórico y epistemológico se corresponde con la práctica exitosa del profesor virtual.

En tal caso, la calidad de la práctica tutorial según Moreno (2007) radica en la integración de los contenidos teóricos con sesiones experenciales, el cual exige un análisis de los productos realizado por los participantes, y posteriormente mediante la iniciativa del tutor, ofrecer un debate de los respectivos aportes individuales o grupales, cerrando el proceso con una respectiva reflexión colectiva

La tutorización integrada por los recursos tecnológicos según Simons, van der Linden y Duffy (citado en Ligorio, Simons y Talamo, 2001) contribuye al "Aprendizaje Nuevo" el cual se caracteriza por nuevos objetivos de aprendizaje, nuevas habilidades de alto nivel, nuevas formas de aprender con énfasis en la participación, nuevos modelos pedagógicos en estudio de casos y resolución de problemas, permitiendo nuevas tendencias en el aprendizaje autorregulado y colaborativo.

No obstante, como bien lo advierte Roca (Citado en Sancho, 2001), aunque se preconiza el establecimiento de una educación abierta y flexible en la formación a distancia, aun disponiendo nuestras universidades de herramientas tecnológicas educativas y novedosas metodologías de aprendizaje, el nivel de interacción docente-estudiante en la tutorización es bajo.

Por otra parte, la modalidad educativa del e-learning supone dentro del ámbito académico de forma generalizada un escepticismo en sus logros, pues compromete la satisfacción en el aprendizaje del discente y el alcance de los objetivos propuestos, generando la preocupación de las universidades e institutos y su inquietud por mejorar de forma progresiva la calidad del servicio educativo a través de los medios tecnológicos, situación que exige replantear los métodos y modelos de enseñanza-aprendizaje en la educación virtual (Seone y García, 2007). 
Aunado a lo anterior, Cabero (citado en Osorio, 2009) señala que es necesario replantear los procesos de enseñanza y aprendizaje a nivel organizacional en las instituciones educativas universitarias, con la presencia de líderes académicos inmersos en la sociedad del conocimiento e involucrados con los principios de gestión como la innovación, la creatividad y la toma de riesgos.

Actualmente, es imprescindible revisar el proceso de tutorización académica universitaria, evaluando la eficacia del e-tutor, con el objeto de evitar la improvisación dentro de las aulas virtuales, sin comprometer la calidad de la enseñanza universitaria y la formación de los estudiantes en las competencias laborales o profesionales que requiere nuestra sociedad, como muy bien lo considera Scheuermann (2003), es obligatorio revisar si los entornos abiertos y flexibles con el apoyo de las TIC nos dirigen a una educación cualitativamente mejor y si es necesario activar nuevos modelos educativos en la modalidad e-learning.

Por lo tanto, el modelo de la enseñanza en línea debe cambiar, aunque las universidades y las distintas instituciones inviertan en soluciones tecnológicas dentro de sus campus, es imprescindible que los ambientes virtuales y la participación del tutor se centre más en el aprendizaje de los estudiantes, para garantizar una formación profesional significativa (Rutkauskiene, Volungeviciene y Kovertaite, 2004).

En contraste, el elearning según Hernández y Tolino (2010) presenta en la realidad educativa algunos inconvenientes, entre los cuales figura la ausencia de la individualización en la enseñanza, por el alto volumen de estudiantes que amerita constante atención, creando un clima de insatisfacción y frustración personal en el estudiante durante su experiencia educativa virtual.

Por otra parte Malbrán (2004) enfatiza que la tutorización electrónica como un soporte educativo para el estudiante demanda una revisión de los materiales de trabajo, las formas de monitorear el desempeño del aprendiz, y la interacción entre el estudiante y el tutor, a fin de asegurar la construcción del conocimiento y la formación de las habilidades.

Dentro del contexto de la situación educativa a nivel superior en Latinoamérica, región que se caracteriza por un incremento del uso de la tecnología, bien amerita de una investigación centrada en la calidad del trabajo académico bajo la modalidad virtual, y en 
su efecto, del seguimiento de su evolución, con el fin de identificar y gerenciar tendencia futuras (Silvio, 2003).

En Venezuela el inicio de la educación virtual se concentró en la promoción de cursos de postgrado, y según La Rocca (2003) el porcentaje de usuarios de postgrado (37\%) se ubica por encima de los usuarios de pregrado (29\%) lo que sugiere la alta demanda y atención en los estudios de postgrado en la modalidad virtual a nivel nacional; en el mismo informe redactado para la UNESCO (Op. cit) se menciona que en el país además de no existir un marco legal regulatorio de la educación universitaria bajo la modalidad virtual, la universidad no cuentan con instrumentos que les permita describir la calidad del servicio educativo a distancia con el apoyo tecnológico.

Específicamente en la Universidad Experimental Libertador Instituto Pedagógico Rural Gervasio Rubio, ubicado en la ciudad de Rubio, estado Táchira, Venezuela, como parte de la atención a una población estudiantil con necesidades de formación en postgrado, los cuales se encuentran actualmente cursando estudios a través de la plataforma educativa moodle, donde se establecen diferentes cursos adscritos a los programas académicos.

No obstante, y de forma generalizada Hope (citado en Silvio, 2006) reconoce la existencia de factores que hacen posible la eficiencia de la educación virtual, factores involucrados en los procesos y las prácticas, los insumos y los recursos diseñados junto con los productos o servicio de aprendizaje, elementos teóricos a considerar en la presente investigación.

En ese orden de ideas, se considera que el proceso educativo a distancia a través de la mencionada plataforma presenta ciertas características en cuanto a la funcionalidad pedagógica, que en cierta forma pesa la participación del tutor, y su papel determina en consecuencia, la calidad del aprendizaje del estudiante de postgrado, con su posible satisfacción en la experiencia educativa durante su respectiva formación en los estudios de postgrado.

En consecuencia, es necesario establecer los factores que permitirán la eficiencia en el proceso que protagoniza el tutor virtual en la formación a distancia dentro del subsistema de educación de postgrado en la UPEL-IPRGR, garantizando la calidad académica de los 
futuros egresados que participan de la experiencia en el aprendizaje bajo la modalidad on line.

En tal sentido, la cuestión planteada como guía para emprender la presente investigación es: ¿Existen factores que contribuyen a la tutorización en línea en la formación de postgrado a través de la plataforma institucional de la UPEL-IPRGR? ¿Qué roles cumple el tutor en el aula virtual en el subsistema de postgrado en la UPEL-IPRGR? ¿Cuáles son las funciones que permite la tutorización virtual en la formación de postgrado?

¿Qué factores permiten el proceso de tutorización y su contribución en la formación de calidad en los cursos de postgrado?

\section{Objetivo General}

Establecer los factores que contribuyen en el proceso de tutorización online en la formación de postgrado en la UPEL-IPRGR

\section{Objetivos Específicos}

Diagnosticar el rol del tutor en el aprendizaje dentro del aula virtual en la formación de postgrado

Describir las funciones del docente que permiten el proceso de tutorización virtual en la formación de postgrado.

Analizar los factores que garantizan el proceso de tutorización en la plataforma virtual

\section{El Rol Del Tutor En La Modalidad De Teleinformación}

La visión de un buen docente universitario es clave como factor que incide en la calidad educativa según Fernández (2009), pues el docente desarrolla un modelo propio enmarcado en un conjunto de acciones que le permite satisfacer las necesidades y expectativas de la formación profesional de los estudiantes, de ahí la importancia apremiante de considerar los roles de un docente universitario virtual.

Los cambios en su defecto originados por la aparición de la educación virtual en los entornos colaborativos según Harasim (2000) plantea el cambio del papel del profesor más 
como un ayudante que como un dador de lecciones, lo que sin duda alguna transforma rotundamente el rol del docente tradicional con respecto al docente virtual, ya que las universidades y el modelo competencial demanda replantear la actuación del docente con su enseñanza tradicional de transmisión de conocimientos (García y Morillas, 2011).

Por lo tanto, es importante la consideración y discusión del rol del tutor virtual, pues según Salmon (2000) el aprendizaje en un entorno on line depende en gran manera del desempeño del tutor como moderador en un aula virtual, y la formación del tutor on line se vincula al grado de motivación de un aprendiz y el desarrollo de sus actividades educativas.

De acuerdo a Mason (1991), con la finalidad de facilitar la enseñanza en línea el docente debe asumir los siguientes roles:

a. Rol organizativo: donde el docente actúa como líder al establecer una agenda de trabajo en la cual mantiene la interacción de los participantes al promover la participación grupal.

b. Rol social: la cual consiste por parte del docente en generar un entorno propicio para el aprendizaje donde de forma abierta el participante se sienta en confianza al expresar sus sentimientos y sensaciones.

c. Rol intelectual: el docente tiene la capacidad de focalizar las discusiones en los puntos cruciales animando a realizar preguntas que permitan emitir los comentarios y aportes significativos.

Con base de los anteriores roles del tutor en línea, el docente debe desarrollar cualidades y habilidades que vislumbren en su nuevo papel de guía y moderador capacidades administrativas, afectivas y académicas que aseguren un desarrollo educativo de calidad en el aula virtual.

a. Por otra parte Bianchino, Marinensi, Medaglia y Ruozzi (2012) enumera como roles del tutor de categoría compleja y multifacética las siguientes competencias:

Moderador y promotor de discusiones en un determinado foro.

b. Facilitador de la formación, especialmente desde el punto de vista de la administración del tiempo y la motivación del aprendiz.

c. Ayudante técnico, en el uso de los recursos que se disponen ante las dificultades 
El anterior autor vincula un buen desempeño del tutor virtual al manejo de los grupos de estudio, como motivador del aprendizaje y apoyo al estudiante en su desenvolvimiento al aprender con las herramientas y recursos educativos que el aula virtual dispone.

En otro orden de ideas, Griffin, Gilchrist, y Thomson. (2009) delinean para el rol del tutor virtual los siguientes principios de las buenas prácticas pedagógicas:

a. Estimar al estudiante: Valora a cada estudiante en su aprendizaje individual considerando sus circunstancias y sus antecedentes.

b. Receptivo a las necesidades educativas del estudiante: Responde al estudiante después de sus intervenciones en el aula virtual, de forma pública y privada.

c. Es motivador y positivo: Se asegura que el estudiante participe en las discusiones reconociendo sus progresos y sus contribuciones.

d. Promueve la autonomía: Asiste al estudiantes contestando sus inquietudes respaldando sus iniciativas en el aprendizaje

De las anteriores prácticas educativas en línea se destaca el papel humano y afectivo del docente, quien se interesa por el desenvolvimiento del estudiante siempre tomando en cuenta sus limitaciones y especiales circunstancias que potencien su desarrollo personal.

\section{Cuadro 1.}

\section{Rol del Tutor Virtual}

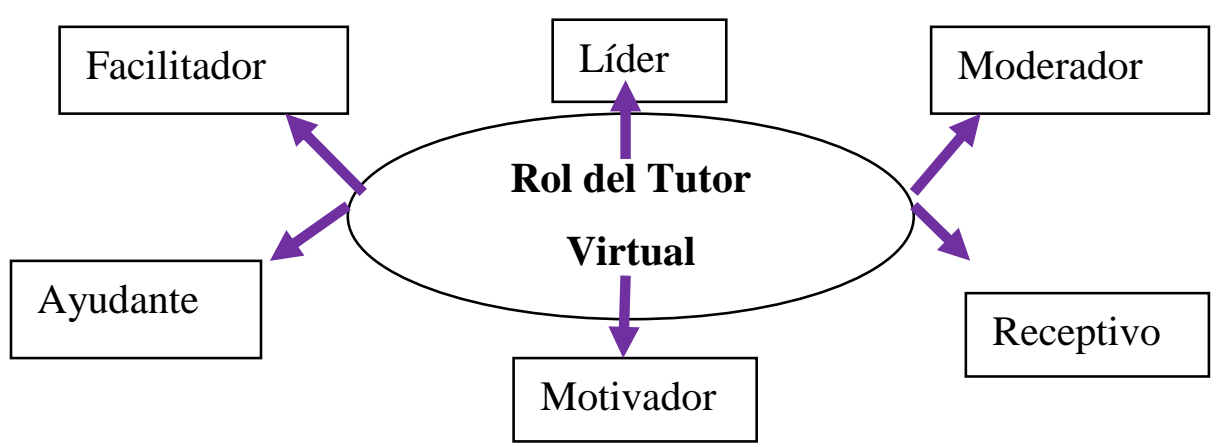

Fuente: El autor 
El anterior cuadro, que muestra en síntesis el rol del tutor virtual según las descripciones de los citados autores, resalta las capacidades o facultades necesarias desplegadas por el docente y que garantizan la satisfacción del estudiante en su experiencia de aprendizaje en el aula virtual.

\section{Funciones del tutor virtual.}

La función del tutor virtual según Lugo (citado en Valverde y Garrido, 2005) debe centrarse más en el aprendizaje como promotor del aprendizaje autónomo desarrollado por los participantes en el aula virtual, y no solo como mero transmisor de la información.

Según García (2001b) las funciones del tutor virtual se despliegan en dos dimensiones, una función orientadora centrado en el aspecto afectivo y una función académica centrada en el ámbito cognitivo.

La función orientadora abarca las siguientes acciones por parte del tutor:

a. Mantener el contacto del participante con la institución

b. Aclarar los objetivos y metas del material educativo

c. Comunicarse de forma personal continuamente con el estudiante a fin de mantener su motivación, desarrollando una comunicación bidireccional formulando preguntas y escuchando al estudiante.

d. Orientar al estudiante en las diversas técnicas de estudio, a fin de desarrollar la redacción y presentación de los trabajos propuestos y su respectiva evaluación.

e. Promover la interacción de los miembros de la comunidad virtual educativa favoreciendo el trabajo colaborativo.

f. Asistir al estudiante en las diferentes dificultades que se presenten en el aprendizaje.

Las recientes líneas donde se especifica las funciones del tutor abarcan su interacción a nivel individual y grupal que permita una experiencia educativa significativa en el aula virtual, logrando la continua participación del estudiante.

Por otra parte, la función académica se encuentra constituida por las siguientes funciones:

a. Función diagnostica: la cual permite indagar los saberes previos, destrezas y 
actitudes del estudiante.

b. Función informativa: presentar los objetivos y contenidos del curso, aclarar los requisitos para abordar el estudio y como utilizar el material didáctico y los diversos recursos disponibles.

c. Función de guía del aprendizaje: Plantear las respectivas metodologías de estudio del curso garantizando la utilidad de los contenidos según los objetivos planteados

d. Función de evaluación: Monitorear el desempeño del estudiante mediante el seguimiento de las actividades realizadas verificando los conocimientos, destrezas y actitudes desarrolladas por el participante.

La función académica del tutor señalada recientemente desde el punto de vista formal traslada el papel del docente tradicional a su desempeño como docente virtual, como administrador y gerente del desarrollo de los aprendizajes.

Por otra parte Adell y Sales (1999) plantean las siguientes funciones del tutor virtual:

a. Diseñar el curso en general, en la cual selecciona los contenidos y los recursos, planifica las diversas actividades.

b. Elaborar los contenidos digitalizados en múltiples formatos que permita la interacción y personalización del material educativo en el aula virtual.

c. Facilitar el aprendizaje de forma directa o indirecta a través de los materiales educativos.

Según Adell y Sales (ob. cit.) la función del tutor virtual se centra en la administración y elaboración de los materiales educativos, destacándose su importancia en el aprendizaje en la web, donde la interacción dependa del contacto del estudiante con los recursos pedagógicos y el docente.

En otro orden de ideas, Llorente (citado en Picón, 2013) menciona cinco funciones básicas del docente en e-learning que a continuación se presentan:

- Función Académica: La cual consiste en informar, aclarar y explicar los contenidos que se presentan, controlar el progreso del participante y monitorear las actividades desarrolladas, establecer la retroalimentación en las actividades del estudiante, verificar si el estudiantes ha logrado los objetivos propuestos, e informar de forma oportuna los resultados alcanzados, formular preguntas con el propósito de determinar el 
conocimiento logrado por el grupo y despejar las dudas, y diseñar actividades que permitan la comprensión del contenido y su aplicación

- Función Social: La responsabilidad del docente se circunscribe en recibir a todos los participantes de forma afectiva, crear los grupos de trabajo, incentivar los comentarios y ampliarlos de los participantes, plantear actividades que faciliten el intercambio de información y promueve un clima de trabajo positivo en el aula virtual.

- Función Organizativa: Esta función se encuentra integrada por planificar e informar sobre las fechas del cronograma de actividades y el criterio de evaluación con su nivel de exigencia en la participación, establecer formas de contacto con el tutor o coordinadores que faciliten la ayuda ante los problemas presentados en la plataforma.

- Función Orientadora: Se destaca en esta función el asesoramiento sobre el estudio en la red, recomendar sobre el trabajo a desarrollar en la red, vigilar que se trabaje a un ritmo apropiado y aconsejar sobre el desarrollo de las actividades.

- Función Técnica: Se trata de solucionar los obstáculos de índole técnico que se presenten al participante

De forma concreta Valverde y Garrido (ob. cit.) establecen las funciones del tutor virtual según el correcto uso de las herramientas web tanto síncronas como asíncronas, especificándolas de la siguiente manera:

a. Funciones del tutor en el uso correcto de las herramientas asíncronas (Correo electrónico y foros de debate):

○ Establecer el contacto directo y personal con el participante.

- Resolver dudas y problemas de forma personalizada.

- Recordar de forma individual la agenda de actividades del curso.

- Orientar de manera dialógica al estudiante.

- Contextualizar los contenidos de acuerdo a la realidad social y cultural.

b. Funciones del tutor en el uso correcto en el uso de las herramientas síncronas (Chat):

○ Orientar las charlas desde un clima social informal que facilite la participación 
- Destacar temas de índole organizativo en lo tocante al cronograma de actividades, estrategias de aprendizaje para el curso, criterios de evaluación objetivos de las tareas y comentarios sobre los trabajos desarrollados.

- Generar temas de conversación ajenas al ámbito académico que permiten la cohesión del grupo.

- Promover temas o cuestiones polémicas que permitan la reflexión para la participación significativa en los foros de discusión.

Ahora bien, en la definición de las dimensiones pedagógicas en el aula virtual Area y Sales (2009) definen en la dimensión del tutor las siguientes funciones a cumplir:

- Habilidades de motivación, refuerzo y orientación sobre los hábitos de estudio a fin de reforzar la participación del estudiante en su desempeño académico.

- Organizar y dinamizar las actividades grupales, donde se evidencie el orden en el trabajo de grupo.

- Adaptación natural de trabajo educativo en los diversos entornos virtuales donde el tutor maneje de forma espontánea la administración de las actividades, su evaluación y monitoreo constante.

- Uso didáctico de los instrumentos telemáticos, donde aplique de forma pedagógica los recursos en los cursos de educación a distancia

A continuación se presenta una tabla que señala las competencias del tutor virtual necesarias para el desarrollo satisfactorio de los estudiantes en el aula virtual según los referentes teóricos consultados en el presente estudio.

Tabla 1. Competencias del Tutor Virtual

\begin{tabular}{|c|c|c|c|c|c|}
\hline \multicolumn{6}{|c|}{ Competencias del Tuto Virtual } \\
\hline $\begin{array}{l}\text { Orienta } \\
\text { Como guía } \\
\text { del proceso } \\
\text { educativo }\end{array}$ & $\begin{array}{l}\text { Comunica } \\
\text { Dialoga } \\
\text { continuamente } \\
\text {,manteniendo } \\
\text { el contacto con } \\
\text { el estudiante }\end{array}$ & $\begin{array}{l}\text { Diagnostica } \\
\text { Revisa las } \\
\text { condiciones } \\
\text { educativas } \\
\text { previas en el } \\
\text { aula virtual }\end{array}$ & $\begin{array}{l}\text { Informa } \\
\text { Mantiene } \\
\text { actualizado } \\
\text { al } \\
\text { participante } \\
\text { de la } \\
\text { actividades y } \\
\text { los } \\
\text { contenidos }\end{array}$ & $\begin{array}{l}\text { Evalúa } \\
\text { Interviene en los } \\
\text { procesos } \\
\text { educativos } \\
\text { informando la } \\
\begin{array}{l}\text { actuación del } \\
\text { participante }\end{array}\end{array}$ & $\begin{array}{l}\text { Diseña } \\
\text { elabora } \\
\text { materiales } \\
\text { Construye de } \\
\text { forma creativa } \\
\text { y original los } \\
\text { contenidos } \\
\text { con formato } \\
\text { interactivo }\end{array}$ \\
\hline
\end{tabular}

Fuente: El autor 
Finalmente, según la anterior tabla las competencias del tutor virtual se centran en sus funciones de guía en el aprendizaje, como un buen comunicador, donde informa oportunamente a los participantes del aula virtual y de forma continua vigila la participación y los productos presentados por el estudiante.

\section{METODOLOGIA}

La presente investigación y su objeto de estudio, el cual es los factores que contribuyen a la tutorización en línea, amerita de la obtención de los datos para su respectiva medición, mediante de las técnicas estadísticas, apoyado del paradigma positivista y un enfoque cuantitativo, donde sea posible realizar la debida abstracción y análisis de los datos para su respectiva discusión y generalización

La presente investigación se enmarca a nivel descriptivo y de campo, y según Silva (2008) una investigación descriptiva usa el método de análisis para caracterizar un fenómeno con la finalidad de señalar sus características y propiedades, y con el respaldo de técnicas de estadísticas para el análisis de la variable se pretende lograr en el estudio la descripción de la labor del docente en el aula virtual a partir de las opiniones de los estudiantes que incursionan en la modalidad semi-presencial.

Ahora bien, el tipo de muestra considerado por el presente estudio, es la muestra probabilística al azar simple, pues según Arias (2006) este tipo de muestra se basa en el procedimiento en el cual todos los individuos poseen en sí la misma probabilidad de ser seleccionados como objeto de unidad de análisis.

En efecto, la fórmula para calcular el tamaño de la muestra, cuando el tamaño de la población es conocido según Palella y Martins (2006) es la siguiente:

$$
\mathrm{n}=\frac{N .}{(N-1) \cdot e^{2}+1}
$$

Donde :

$\mathrm{n}=$ Tamaño de la muestra

$\mathrm{N}=$ Total de elementos que integran la población 
e $=$ error de estimación de $9 \%$

Entonces el tamaño de la muestra se procede a calcular, quedando:

$$
\mathrm{n}=\frac{200}{(200-1) \cdot(0,09)^{2}+1}=\frac{200}{2,61}=77,6 \approx 77 \text { estudiantes }
$$

Según Pallela y Martins (ob. cit.) el diseño de la investigación responde al problema de investigación mediante una estrategia que asume el investigador, siendo el diseño no experimental, ya que no se requiere según los objetivos planteados la manipulación de la variable.

En cuanto a la variable de estudio de la presente investigación, ésta se descompone en una serie de factores que estadísticamente de forma conjunta comparte en su medición, una varianza en común, y a su vez se observa ciertas correlaciones que le brinda una regularidad en el comportamiento de los datos, el que hace posible la aplicación del método clásico de análisis de factores (Bolívar, 2002).

El Análisis factorial tiene como propósito según Aiken (2003) busca reducir el número de variables en un grupo de medidas tomando en cuenta la correlación (traslape) entre ellas, y en consecuencia de acuerdo a la propuesta de esta técnica se realizará la correlación entre los ítems, para cruzar la asociación entre los indicadores propuestos en el presente estudio y obtener los factores asociados en el papel que desempeña el tutor virtual según las valoraciones de los encuestados.

Como el objetivo principal del estudio en la recolección de la información es acudir a la mayor audiencia posible, digno de los estudios cuantitativos, la técnica escogida para tal fin es la encuesta y el instrumento el cuestionario, y según Shaughnessy, Zechmeister. y Zechmeister (2007) el cuestionario es el instrumento principal de investigación, porque tiene el poder científico de medir variables, y la calidad de la medición depende de la calidad del cuestionario.

El criterio para la selección de los ítems después de la prueba piloto de 50 ítems, se escogió con la condición de que el valor de su varianza obtenida por cada ítem fuera menor de 2,5, y según esta condición fueron seleccionados los 21 ítems del cuestionario definitivo. 


\section{RESULTADOS}

Después de la recolección y procesamiento de los datos según Sabino (1992) es necesario el análisis crítico de la información, en el cual se procede a sistematizar y sintetizar los resultados, con la finalidad de arribar a las conclusiones globales de acuerdo a los datos obtenidos.

En el análisis de factores sobre los resultados obtenidos se realizó la prueba de sedimentación para la obtención respectiva de los factores o componentes iniciales a considerar.

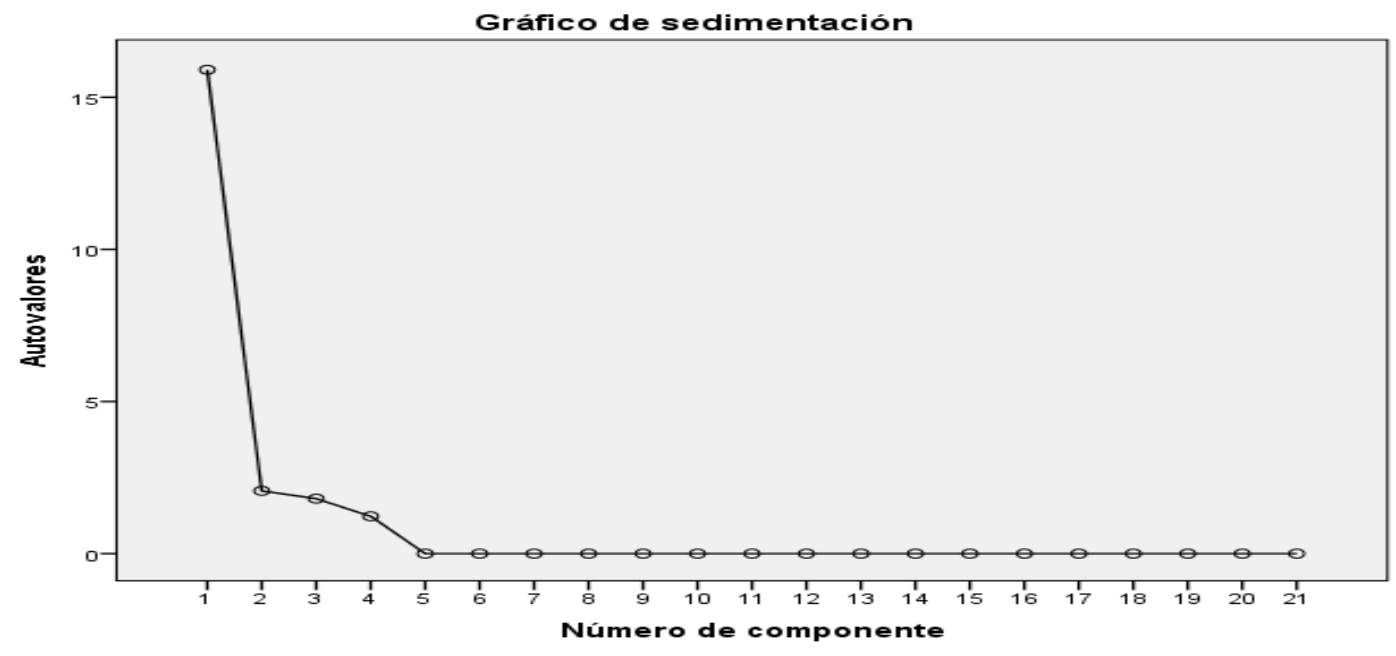

Grafico 1. Número de componentes de los ítems

Según el grafico de sedimentación se sugiere considerar cuatro (4) factores como componentes principales como técnica exploratoria para examinar la estructura de los datos en la matriz y lograr la reducción de los datos, y a continuación la siguiente tabla revela la estructura de la matriz sin rotación. 
Tabla 2. Matriz de Factores sin rotar

Matriz de componentes ${ }^{\mathrm{a}}$

\begin{tabular}{|c|c|c|c|c|}
\hline & \multicolumn{4}{|c|}{ Componente } \\
\hline & 1 & 2 & 3 & 4 \\
\hline $00001{ }^{\text {VAR }}$ & ,945 & , 168 &, 007 & ,282 \\
\hline $00002^{\text {VAR }}$ & ,876 & ,395 & 206 & ,185 \\
\hline VAR & 990 & & ,133 & \\
\hline VAR & 836 &, $0<7$ & & , \\
\hline 00004 & (1) & 隹 &, 121 &, 310 \\
\hline $00005^{\text {VAR }}$ & ,798 &, 575 &, $145^{-}$ &, $107^{-}$ \\
\hline $00006^{\text {VAR }}$ & ,386 & , 074 & ,917 &, 074 \\
\hline $00007^{\text {VAR }}$ & ,987 &, 030 &, $032^{-}$ & ,153 \\
\hline $00008^{\text {VAR }}$ & ,806 &, 565 &, 078 & ,158 \\
\hline 00009 & ,806 & ,129 &, 474 & ,329 \\
\hline $00010^{\text {VAR }}$ & ,905 & ,322 & ,269 & ,063 \\
\hline 00011 & ,979 &, 106 &, 082 & , 155 \\
\hline $00012^{\text {VAR }}$ & ,932 & , 126 & ,269 & ,208 \\
\hline $00013^{\text {VAR }}$ & 630 &, $210^{-}$ & ,099- & ,741 \\
\hline &, 851 & ,510 & ,116 & , 047 \\
\hline $00015^{\text {VAR }}$ & 662 & ,267 & ,618 &, 330 \\
\hline 00016 & ,972 &, 063 & ,064 & ,218 \\
\hline $00017^{\text {VAR }}$ & ,973 &, 090 & ,097 & , 190 \\
\hline $00018^{\text {VAR }}$ & ,928 & ,361 & ,068 & ,062 \\
\hline $00019^{\text {VAR }}$ & ,929 & ,324 &, 127 &, 122 \\
\hline $00020^{\text {VAR }}$ & ,863 &, 502 &, 025 & ,046 \\
\hline $00021^{\text {VAR }}$ & ,969 & ,088 & ,216 & 078 \\
\hline
\end{tabular}

Método de extracción: Análisis de componentes principales.

a. 4 componentes extraídos 
Es de resaltar que se establece según la observación de las correlaciones de los factores de los componentes número 3 y 4 , no se tomarán en cuenta porque los pesos factoriales no son representativos en comparación con los dos primeros componentes ya analizados

Según la anterior tabla se tomará en cuenta el factor I (Factor general) relacionado con el factor II (Factor Bipolar), al primer componente se le llamará habilidades académicas y al segundo componente habilidades sociales del e-tutor, y de los dos primeros componentes mantiene la más alta correlación o pesos factoriales los ítems 5 y 8 , esto se interpreta como que el tutor con su habilidad académica como guía asiste al estudiante ante sus inquietudes, la cual está ligada a su habilidad social como motivador en la comunicación bidireccional con sus estudiantes en el aula virtual.

Tabla 3 Matriz de Factores con la primera rotación (Varimax)

\begin{tabular}{|c|c|c|}
\hline & \multicolumn{2}{|c|}{ Componente } \\
\hline & 1 & 2 \\
\hline VAR & ,896 & ,405 \\
\hline $00002^{\text {VAR }}$ & ,389 & ,829 \\
\hline $00003^{\text {VAR }}$ & ,751 & ,518 \\
\hline $00004^{\text {VAR }}$ & ,507 & ,856 \\
\hline $00005^{\text {VAR }}$ & ,331 & ,941 \\
\hline $00006^{\text {VAR }}$ & ,250 & ,036 \\
\hline 00007 VAR & ,665 & ,572 \\
\hline $00008^{\text {VAR }}$ & ,221 & 893 \\
\hline 00009 VAR & 604 & ,391 \\
\hline $00010^{\text {VAR }}$ & ,842 & ,210 \\
\hline $00011^{\text {VAR }}$ & ,851 & ,480 \\
\hline $00012^{\text {VAR }}$ & ,868 & ,465 \\
\hline $00013^{\text {VAR }}$ & ,325 & 153 \\
\hline 00014 & ,321 &, 874 \\
\hline
\end{tabular}




\begin{tabular}{|l|c|c}
00015 VAR &, 154 &, 487 \\
00016 VAR &, 843 &, 512 \\
VAR &, 830 &, 467 \\
00017 VAR &, 873 &, 229 \\
00018 VAR &, 810 &, 231 \\
00019 VAR &, 931 &, 071 \\
00020 VAR &, $\mathbf{8 2 1}$ &, $\mathbf{5 0 2}$ \\
\hline \multicolumn{2}{|l}{ VAR } &
\end{tabular}

Ahora bien en la primera rotación de la matriz, se vislumbra por la relación de los pesos factoriales la habilidad académica del tutor virtual como facilitador de la formación y su habilidad en el uso didáctico de los materiales educativos digitalizados.

\section{DISCUSIÓN}

En primer lugar con respecto a las roles del tutor virtual en el aula virtual del Pedagógico de Rubio, específicamente en la formación de postgrado que se observa la dificultad del docente al focalizar las discusiones en los diversos foros restando en consecuencia la participación significativa de los estudiantes.

En consecuencia es primordial la formación del profesorado en su intervención como e-tutor, concretamente en su participación como académico, donde tome la iniciativa en guiar las intervenciones de los estudiantes enfocando y reenfocando las discusiones según las temáticas abordadas de forma asíncrona o síncrona.

En segundo lugar y ahora con respecto a las funciones del tutor virtual, es necesario reorientar la habilidad del docente en facilitar el aprendizaje del estudiante por medio de los materiales educativos colocados en el aula virtual, ya que los mismos guían al estudiante en el desarrollo de las actividades educativas propuestas.

En tal caso, se recomienda al docente tutor elaborar y disponer los materiales educativos digitalizados en el aula según los principios teóricos del diseño instruccional, de tal forma que los materiales se adapten a la audiencia según sus necesidades educativas y el estilo de aprendizaje. 
Finalmente se relaciona la habilidad académica del tutor como facilitador de la formación en el aula virtual y la habilidad social como motivador del aprendizaje, siempre que cuente con la función de organizador y administrador del aula virtual, con la disposición de idóneas actividades y recursos educativos.

\section{REFERENCIAS}

Adell, J, y Sales, A. (1999). El profesor on line: Elementos para la definición de un nuevo rol docente. Edutec. Disponible: http://especializacion.una.edu.ve/fundamentos/paginas/adell.pdf $\quad[$ Consulta:3 de marzo de 2014 ]

Aiken, L. (2003). Test psicológicos y evaluación. Editorial Pretince Hall. Undécima Edición. México.

Area, M. y Adell, J. (2009). eLearning: Enseñar y aprender en Espacios Virtuales. Disponible: http://tecedu.webs.ull.es/textos/eLearning.pdf [Consulta: 5 de febrero de 2014]

Arias, F. El proyecto de investigación. 5ta Edición. Editorial Episteme. Caracas.

Bianchino, C. Marinensi, G, Medaglia, C. y Ruozzi, E. (2012). The role of e-tutors in the elearning training paths: the experience of the Italian Revenue Agency. Journal of Elearning and Knowledge Society. V. 8 , Num 1 , 23-31 Disponible: www.editlib.org/d/43386/ [Consulta: 13 de agosto de 2014]

Bolívar, C. (2002). Instrumentos de investigación educativa. Editorial CIDEG. Barquisimeto. Venezuela

Fernández, R. (2009). Factores antecedentes en el uso Entornos Virtuales de Formación y su efecto sobre el Desempeño Docente. Tesis Doctoral. Universidad Politécnica de Valencia. Disponible: http://revistavirtual.ucn.edu.co/ / [Consulta: 13 de agosto de 2014]

García, L. (2001a). La educación a distancia. De la teoría a la práctica. Barcelona: Ariel.

García, L. (2001b). Educación a Distancia. Ayer y hoy. Universidad Nacional de Educación a Distancia. Disponible:

García, M. y Morillas, L. (2011). La planificación de evaluación de competencias en educación superior. Nuevos Desafíos en la formación del Profesorado. Revista Electrónica Interuniversitaria de Formación del Profesorado. REIFOP. N. 36. España. 
Disponible: $\quad$ www.aufop.com/aufop/uploaded_files/revistas/130493241710.pdf [Consulta: 22 de marzo de 2014]

Griffin, T., Gilchrist, A. y Thomson, R. (2009). Role of the online tutor in a large enrolment unit. University of the Western Sidney. [Articulo en Línea]. Disponible: http://red-u.net/redu/documentos/vol11_n2_completo.pdf [Consulta: 10 de junio de 2014]

Harasim, L. (2000) Redes de Aprendizaje. Barcelona: Gedisa.

Hernández,. M. y Tolino A. (2010). Contextos no presenciales y tutorial. El tutor elearning. Universidad de Murcia. Disponible: http://ticemur.fintegra.org/comunicaciones/virtuales/03.pdf [Consulta: 14 de abril 2014]

La Rocca, R (2003 ). Diagnóstico de la Educación Superior Virtual en Venezuela. En La Educación Superior Virtual en América Latina y el Caribe. Instituto Internacional para la Educación Superior en América Latina y el Caribe. UNESCO. Disponible: dialnet.unirioja.es/descarga/articulo/3945704.pdf [Consulta: 7 de julio 2014]

Ligorio, M, Simons, P. y Talamo, A. (2001). Synchronic Tutoring of Virtual Community . [Documento en Linea] Disponible: http://webcache.googleusercontent.com/search?q=cache:IsivE88HYVgJ:www.resear chgate.net/profile/Beatrice_Ligorio/publication/27690333_Synchronic_tutoring_of_a _virtual_community/links/00b7d52056db970fb5000000.pdf+\&cd=1\&hl=es\&ct=clnk $\& \mathrm{gl}=$ ve [Consulta: 18 de febrero de 2014]

Malbrán, M. (2004). La Tutoría en el nivel universitario. Universidad Nacional de la Plata. Buenos Aires. Revista de Informática Educativa y Medios Audiovisuales. Vol 1 (1). Pag. 5-11. Disponible: http://sedici.unlp.edu.ar/bitstream/handle/10915/21263/Documento_completo.pdf?se quence $=1$ [Consulta: 26 de abril de 2014]

Mason, R. (1991) Moderating educational computer conferencing. Deosnews. Vol 1 . No. 19

Disponible: http://www.researchgate.net/publication/240122602_Moderating_educational_compu ter_conferencing [Consulta: 11 de agosto 2014]

Medina, Y., Rico, D. y Rico, M. (2011). Calidad en la función tutorial para la gestión en entornos virtuales. Disponible: http://www.educacioneningenieria.org/index.php/edi/article/view/127 [Consulta: 25 de abril de 2014]

Moreno, Q. (2007). Organización y dirección de los centros educativos innovadores. Editorial McGraw-Hill. Madrid 
Montserrat, S. y Gisbert, M. (2007). "E-tutoría: uso de las tecnologías de la información y comunicación para la tutoría académica universitaria". Teoría de la educación. Educación y cultura en la sociedad de la información, 8 (2), 31-54. Disponible: www.usal.es/ teoriaeducacion/rev...08.../n8_02_sogues_gisbert_isus.pdf [Consulta: 13 de junio de 2014]

Osorio, B. (2009). La figura del e-moderador en el entorno e-learning. Universidad Rey Juan Carlos [Documento en Línea] Disponible: www.raco.cat/index.php/RUSC/article/download/140243/191429 [Consulta: 14 de marzo de 2014]

Pallela, S. y Martins, F. (2006). Metodología de la investigación cuantitativa. FEDUPEL. Caracas.

Pérez, J. (2003). Internautas y náufragos. La búsqueda del sentido en la cultura digital. Editorial Trotta.

Picón, M. (2013). Guía de Tutorización e-learning. Orientaciones básicas sobre la docencia en modalidad de tele formación. Instituto Andaluz de Administración Pública. [Documento en Línea] Disponible: http://www.juntadeandalucia.es/agenciadecalidadsanitaria/formacionsalud/export/site s/default/galerias/aportesDocumentos/pildora/1371807956935.pdf [Consulta: 26 de junio de 2014]

Rutkauskiene, D., Volungeviciene, A. y Kovertaite, V. ( 2004). Tutoring Adults Online. Distance Education Centre at Kaunas University of Technology, Lithuania Disponible: http://ben.upc.es/butlleti/innsbruck/416-225.pdf [Consulta: 25 de febrero de 2014]

Sabino, C. (1992). El proceso de investigación. Editorial Panapo. Caracas.

Salas, Y. (2012). Cibercultura y Educación. Revista Dia-logos Año 7. No. 11 . Costa Rica. Disponible: [Consulta: 14 de marzo de 2016]

Salmon, G. (2000). E-Moderating: The key to teaching and learning on line, Kogan Page, London.

Sancho, J. (2001). Para una tecnología educativa. Cuadernos para el análisis. Editorial Horsori. Barcelona.

Scheuermann, F. (2003) Una vía hacia el futuro de la educación. Enseñanza a distancia y e-learning. Formación Profesional. Revista Europea. N. 27 Disponible: www.cedefop.europa.eu/files/27-es.pdf [Consulta: 27 de marzo de 2014]

Seone, A. Garcia. F. , Bosom, A, Fernández, E., y Hernández , A. (2006). Tutoring on line as quality guarentee on learning base lifelong learning. Definition, modalities, 
methodology, competence and skills. Universidad de Salamanca. Disponible: ceurws.org/Vol-186/05.pdf [Consulta: 25 de agosto de 2014]

Seone, A. y García, F(2007). Los Orígenes del Tutor: Fundamentos Filosóficos y Epistemológicos de la Monitorización para su aplicación a contextos de E-learning. En Tutoría Virtual y e-moderación en red. Revista Electrónica Teórica de la Educación y Cultura en la Sociedad de la Información. Monográfico. Volumen 8. . Disponible: campus.usal.es/ teoriaeducacion/rev...08.../monografico_n8_02.pdf [Consulta: 21 de marzo de 2014]

Shaughnessy, J. Zechmeister, E. y Zechmeister, J. (2007). Métodos de Investigación en Psicología. Editorial McGraw-Hill. Séptima Edición. México.

Silva, J (2008). . Metodología de la Investigación. Ediciones CO-BO. Caracas.

Silvio, J. (2003). Tendencias de la Educación Superior Virtual en América Latina y el Caribe. IESALC. En La Educación Superior Virtual en América Latina y el Caribe. Instituto Internacional para la Educación Superior en América Latina y el Caribe. UNESCO. Disponible: www.iesalc.unesco.org.ve/index.php?option... [Consulta: 12 de febrero de 2014]

Silvio, J. (2006). Hacia una educación virtual de calidad, pero con equidad y pertinencia. Revista de Universidad y Sociedad del Conocimiento. Vol. 3 No.1. Disponible www.raco.cat/index.php/RUSC/article/download/49336/50225 [Consulta: 27de marzo de 2014]

UNESCO (1998). La Educación Superior en el siglo XXI. Conferencia mundial de la Educación Superior. [Documento en Línea] Disponible: http://www.unesco.org/education/educprog/wche/declaration_spa.htm [Consulta: 15 de febrero de 2014]

Valverde, J. y Garrido, M.(2005). La función tutorial en entornos virtuales de aprendizaje: comunicación y comunidad. Revista Latinoamericana de Tecnología Educativa. Vol. 4 pp. 153-167. Disponible: dialnet.unirioja.es/descarga/articulo/1303758.pdf [Consulta: 13 de abril de 2014] 